\title{
The effects of an irrelevant intertrial task on pattern discrimination in rats with hippocampal damage*
}

\author{
GAY B. ALEXANDER, BELINDA BROOME, and LARRY W. MEANS \\ East Carolina University, Greenville, North Carolina 27834
}

\begin{abstract}
Rats sustaining either hippocampal lesions or sham operations were compared on maze activity and visual pattern discrimination in a Y-maze. On the discrimination problem, approximately one-half of the brain-damaged and sham-operated rats were trained with an irrelevant runway task interposed during the intertrial intervals. The rats with hippocampal lesions were found to be more active in the maze than the controls, but hippocampally damaged rats showed no evidence of a discrimination deficit, with or without distraction.
\end{abstract}

Attempts to produce the dramatic amnestic syndrome found in humans after damage to the hippocampus (Milner, 1970) have had mixed results in animal studies. Most studies have shown that infrahumans with hippocampal damage have no difficulty on tasks which do not involve response inhibition, but perform poorly on such tasks as reversals, passive avoidance, extinction, habituation, and spontaneous alternation (Douglas, 1967; Kimble, 1968; Altman, Brunner, \& Bayer, 1973), all of which involve inhibition of some response.

In analyzing the deficit produced by bilateral hippocampal damage in humans, Milner (1970) found that a patient retained a number for $15 \mathrm{~min}$ only by constant verbal rehearsal. If the $S$ were engaged in a conversation, he could recall neither the number nor the train of thought by which he retained it for that period.

Paralleling this procedure, a distracting stimulus has been introduced during learning trials in several animal studies. It was found that the presentation of tactile (Wickelgren \& Isaacson, 1963) and visual stimuli (Raphelson, Isaacson, \& Douglas, 1965) do not disrupt the runway performance of hippocampally damaged rats, and presentation of food does not disrupt performance of a delayed-response task by monkeys with hippocampal lesions (Drachman \& Ommaya, 1964).

Recently, there have been two reports of deficient performance in animals with hippocampal lesions receiving intertrial interval (ITI) distraction. Walker and Means (1973) found that interposing a CRF task during the ITI temporarily disrupts performance on a single-alternation task by rats, and Jarrard (1973) found that forced running in an activity wheel during the ITI disrupts single alternation in the $\mathrm{Y}$-maze by rats.

The present study is an attempt to evaluate further the effects of ITI distraction on the performance of hippocampally damaged rats. Specifically, the study

\footnotetext{
*This paper is based on a thesis submitted by Gay B. Alexander to East Carolina University in partial fulfillment of the requirements for an MA. The research was supported in part by a grant from North Carolina Mental Health to Larry W. Means. The authors thank Patricia Willis and Henry Hooks for their assistance. Reprint requests should be sent to Larry W. Means, Department of Psychology, East Carolina University, Greenville, North Carolina 27834.
}

examines the effects of subjecting hippocampally damaged rats to an unsolvable straight-alley task during the ITIs of acquisition of a visual-pattern discrimination task.

\section{METHOD}

\section{Subjects}

Fifty-three 120-day-old Long-Evans male rats from the East Carolina University colony served as Ss. They were housed individually in a 14-h light, 10-h dark environment and run during the light hours of the cycle. They were maintained at $85 \%$ of their ad lib body weights and given free access to water.

\section{Apparatus}

A two-choice Y-maze, described elsewhere (Means et al, 1973), was used for the discrimination task. The startbox and choice point were painted gray. One goalbox had vertical black and white stripes, while the other had horizontal stripes.

The disruption task was conducted in a gray runway measuring $11 \mathrm{~cm}$ wide, $10 \mathrm{~cm}$ high, and $130 \mathrm{~cm}$ long. At each end of the runway was a box $25 \mathrm{~cm}$ long that was separated from the runway by guillotine doors.

\section{Design}

On the discrimination problem, a 2 by 2 design with Ss assigned according to surgery condition, hippocampal lesion or sham operation, and acquisition condition, disruption or nondisruption, was used. The four groups were: hippocampal lesion, ITI disruption (H-D); hippocampal lesion, no disruption (H-ND); sham operation, ITI disruption (S-D); and sham operation, no disruption (S-ND). Trials to criterion and errors during the first 100 trials were recorded as dependent variables. Also, the number of maze sections entered each minute for 3 min during maze adap tation was recorded.

\section{Surgery}

Surgery was performed using sodium pentobarbital for anesthesia. Each $\mathrm{S}$ also received $.2 \mathrm{cc}$ Bicillin. Bilateral anodal dc hippocampal lesions were produced by applying current at three placements on each side of the brain, one anterior and two posterior. Control Ss received sham operations, which involved lowering the electrode to the same placements but passing no electrical current.

\section{Procedure}

Ss were handled for 4 consecutive days prior to surgery and allowed 2 weeks to recuperate from surgery. Food deprivation and handling began immediately following recovery.

When all Ss reached $85 \%$ of their ad lib body weights, 4 days 

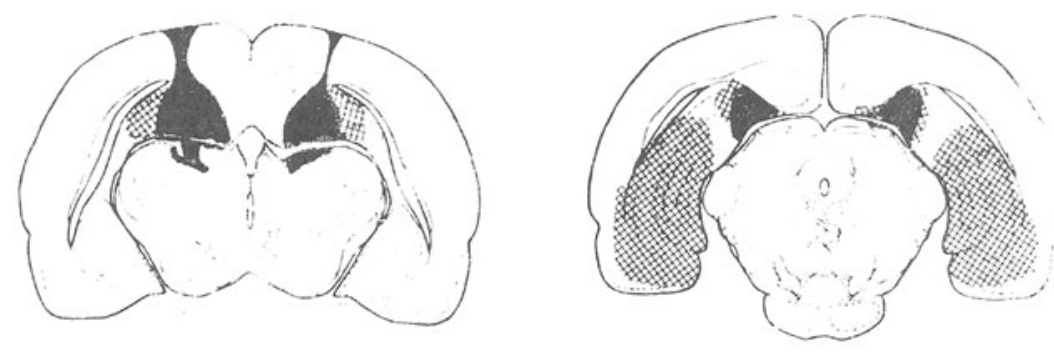

Fig. 1. Reconstructions of the largest and smallest lesions.

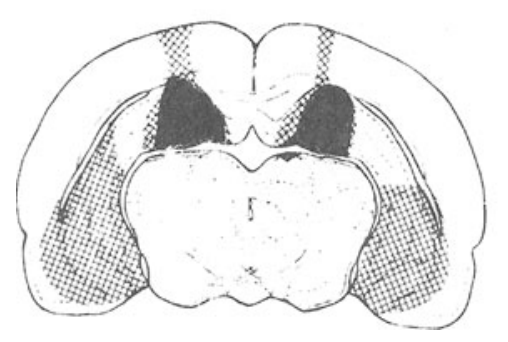

of maze adaptation began. For the adaptation trials, the patterned goalboxes were randomly alternated from left to right and each goalbox contained reinforcement (four Noyes $45-\mathrm{mg}$ food pellets). Each $\mathrm{S}$ remained in the maze at least $3 \mathrm{~min}$, during which activity measures were taken. The $\mathrm{S}$ was removed from the maze after all the pellets had been eaten or after a total of 5 min, whichever occurred first.

On the fifth day, discrimination training began. Each $S$ received four trials per day with an ITI of $1 \mathrm{~min}$. Criterion for learning the task was 14 out of 16 correct choices. Each animal received at least 100 trials. Correct choices were rewarded with four Noyes 45-mg food pellets. The side of the reinforced choice was varied semirandomly, with the restriction that the correct choice be on the left and right sides an equal number of times within each block of eight trials.

Each $\mathrm{S}$ was allowed to make only one choice per trial. Criterion for a choice was that the $S$ have all four paws inside the goalbox. If a $\mathrm{S}$ failed to make a choice within $3 \mathrm{~min}$ after leaving the startbox, that trial was terminated. If a $S$ failed to make a choice on two consecutive trials, the remaining trials for the day were terminated. Any $S$ which failed to complete its trials on 2 consecutive days was dropped from the study.

During the ITI, Ss in Groups H-D and S-D were lifted from the goalbox of the discrimination maze and placed randomly at one of the two ends of the runway, where they were given one trial. In the runway, reinforcement (four Noyes $45-\mathrm{mg}$ food pellets) was provided at the other end on a random $50 \%$ schedule so that there was no solvable pattern. Ss in Groups H-ND and S-ND were lifted from the goalbox of the discrimination maze and placed in their home cages during the ITI.

\section{RESULTS}

Histological examination revealed that 5 animals had improperly placed lesions. These animals were dropped from the study. Eleven other animals were dropped from the study for failing to respond on the discrimination task. The histological and behavioral results reported are thus based on $9 \mathrm{H}-\mathrm{D}, 7 \mathrm{H}-\mathrm{ND}, 12 \mathrm{~S}-\mathrm{D}$, and $9 \mathrm{~S}-\mathrm{ND}$ animals. The animals with lesions showed a range of 23\%-67\% total damage to the hippocampus (see Fig. 1). All lesions included some damage to the corpus callosum and to the posterolateral and entorhinal cortex. Several lesions also included a small amount of damage to the lateral thalamus and medial geniculate body.

The means of the activity measures are shown in Table 1. Examination of the means and the results of a three-way analysis of variance (Lesion by Day by Minutes) showed that hippocampally damaged Ss were significantly more active than sham-operated Ss $(F=$ $10.17, \mathrm{df}=1 / 35, \mathrm{p}<.05)$ and that there was a decrease in activity over days $(\mathrm{F}=3.62, \mathrm{df}=3 / 105, \mathrm{p}<.05)$ and over minutes $(F=23.39, \mathrm{df}=2 / 70, \mathrm{p}<.01)$. The analysis revealed no significant interaction effects.

Table 2 shows the results of the discrimination task. A two-way analysis of variance (Surgery Group by Disruption vs Nondisruption) revealed no significant differences in the number of trials it took to learn the task. A similar analysis showed there were no significant differences with respect to the total number of errors made during the first 100 trials. Thus, the present study offered no evidence for differences among the four groups on acquisition of the visual pattern discrimination.

A correlational analysis was done to determine if any lesion-behavior relationships existed. Correlations between two behavioral measures, trials to criterion and total activity, and the lesion measures, total

Table 1

Mean Activity Scores on Four Consecutive Days for Hippocampally Damaged and Sham-Operated Ss

\begin{tabular}{|c|c|c|c|c|c|}
\hline \multirow{2}{*}{$\begin{array}{l}\text { Surgery } \\
\text { Condition }\end{array}$} & \multicolumn{4}{|c|}{ Days } & \multirow[b]{2}{*}{ Mean } \\
\hline & 1 & 2 & 3 & 4 & \\
\hline $\begin{array}{l}\text { Hippocampal } \\
\text { Lesion }\end{array}$ & 22.00 & 22.07 & 21.75 & 20.68 & 21.62 \\
\hline $\begin{array}{l}\text { Sham- } \\
\text { Operated }\end{array}$ & 20.62 & 16.82 & 15.81 & 15.95 & 17.30 \\
\hline
\end{tabular}


Table 2

Mean Number of Errors in First 100 Trials and Mean Number of Trials to Criterion for Hippocampally Damaged and ShamOperated Ss Under Disruption and Nondisruption Conditions

\begin{tabular}{lccccc}
\hline & \multicolumn{2}{c}{ Disruption } & & \multicolumn{2}{c}{ Nondisruption } \\
\cline { 2 - 3 } \cline { 6 - 7 } Surgery Condition & Errors & Trials & & Errors & Trials \\
\hline Hippocampal Lesion & 36.6 & 92.0 & & 38.4 & 98.8 \\
Sham-Operated & 30.0 & 87.0 & & 35.6 & 86.1 \\
\hline
\end{tabular}

hippocampal damage, bilateral hippocampal damage, dorsal hippocampal damage, ventral hippocampal damage, total lesion size, and posterolateral and entorhinal cortex damage, were computed. The only significant correlations were between bilateral hippocampal damage and activity $(\mathrm{r}=.55, \mathrm{p}<.05)$ and dorsal hippocampal damage and activity $(\mathrm{r}=.67$, $\mathrm{p}<.01)$. Thus, the amount of hippocampal damage, particularly dorsal hippocampal damage, seems to be related to an increase in activity.

\section{DISCUSSION}

The finding that hippocampally damaged animals are significantly more active than sham-operated controls in a maze is consistent with the results of other studies (Means, Leander, \& Isaacson, 1971; Teitelbaum \& Milner, 1963). In addition, the significant correlations of dorsal and bilateral hippocampal damage with activity indicate that hippocampal damage is probably responsible for the increased activity.

Analysis of the discrimination measures show that hippocampally damaged animals have no difficulty learning a simple discrimination, a finding which is well documented in maze discrimination tasks (Mishkin, 1954; Silveira \& Kimble, 1968; Means \& Douglas, 1970). The results also reveal that an ITI runway task does not significantly disrupt acquisition of pattern discrimination in a Y-maze by hippocampally damaged rats. The failure to show a significant disruption effect is consistent with several studies (Raphelson, Isaacson, \& Douglas, 1965; Wickelgren \& Isaacson, 1963; Drachman \& Ommaya, 1964). The results, however, seem inconsistent with studies in which a deficit was reported (Jarrard, 1973; Walker \& Means, 1973).

Studies in which a performance deficit was found and attributed to disruption involved either an operant single-alternation task with CRF disruption (Walker \& Means, 1973) or a Y-maze single-alternation task with activity wheel disruption (Jarrard, 1973). Despite their differences, both studies involved single-alternation tasks which required Ss to use information from the immediately preceding trial in order to respond appropriately. Also, both studies used disruption tasks which involved making some response rather than merely perceiving a stimulus. Studies which have tested the disruptive effects of environmental stimuli (Drachman \& Ommaya, 1964; Raphelson et al, 1965; Shapiro, Gol, \& Kellaway, 1965; Wickelgren \& Isaacson, 1963) indicate that stimuli are not sufficient to impair the performance of hippocampally damaged animals. It is interesting to recall that distractors which have been found to disrupt the short-term memory of humans have involved the performance of a verbal response by the patient (Milner, 1970).

The present study attempted to disrupt hippocampally damaged animals with a motor activity (straight alley task) but failed to produce a deficit. This failure, seen in view of the studies by Walker and Means (1973) and Jarrard (1973), would appear to suggest that motor activity disruption must be used in conjunction with a learning task which requires specific memory of trial-to-trial events. Performance on tasks in which the associative strength gained over all previous trials is adequate to make correct responses apparently is not subject to ITI disruption in Ss with hippocampal damage.

\section{REFERENCES}

Altman, J., Brunner, R. L., \& Bayer, S. A. The hippocampus and behavioral maturation. Behavioral Biology, 1973, 8, 557-596.

Douglas, R. J. The hippocampus and behavior. Psychological Bulletin, 1967, 67, 416-442.

Drachman, D. A., \& Ommaya, A. K. Memory and the hippocampal complex. Archives of Neurology, 1964, 10, 411-425.

Jarrard, L. E, Effects of interpolated activity on short-term retention in rats with hippocampal lesions. Paper presented at the Psychonomic Society meetings, St. Louis, November 1973.

Kimble, D. P. The hippocampus and internal inhibition. Psychological Bulletin, 1968, 70, 285-295.

Means, L. W., \& Douglas, R. J. Effects of hippocampal lesions on cue utilization in spatial discrimination in rats. Journal of Comparative \& Physiological Psychology, 1970, 73, 254-269.

Means, L. W., Huntley, D. H., Anderson, H. P., \& Harrell, T. H. Deficient acquisition and retention of a visual-tactile discrimination task in rats with medial thalamic lesions. Behavioral Biology, 1973, 9, 435-450.

Means, L. W., Leander, J. D., \& Isaacson, R. L. The effects of hippocampectomy on alternation behavior and response to novelty. Physiology \& Behavior, 1971, 6, 17-22.

Milner, B. Memory and the medial temporal regions of the brain. In K. H. Pribram and D. E. Broadbent (Eds.), Biology of memory. New York: Academic Press, 1970.

Mishkin, M. Visual discrimination performance following partial ablations of the temporal lobe: II. Ventral surface vs hippocampus. Journal of Comparative \& Physiological Psychology, 1954, 47, 187-193.

Raphelson, A. C., Isaacson, R. L., \& Douglas, R. J. The effect of distracting stimuli on the runway performance of limbic damaged rats. Psychonomic Science, 1965, 3, 483-484.

Shapiro, W. G., Gol, A., \& Kellaway, P. Acquisition, retention, and discrimination reversal after hippocampal ablation in monkeys. Experimental Neurology, 1965, 13, 128-144.

Silveira, J. M., \& Kimble, D. P. Brightness discrimination and reversal in hippocampally lesioned rats. Physiology \& Behavior, 1968, 3, 625-630.

Teitelbaum, H., \& Milner, P. Activity changes following partial hippocampal lesions in rats. Journal of Comparative \& Physiological Psychology, 1963, 56, 284-289.

Walker, D. W. \& Means, L, W. Single-alternation performance in rats with hippocampal lesions: Disruption by an irrelevant task interposed during the intertrial interval. Behavioral Biology, 1973, 9, 93-104.

Wickelgren, W. O., \& Isaacson, R. L. Effect of the introduction of an irrelevant stimulus on runway performance of the hippocampectomized rat. Nature, 1963, 200, 48-50. 